\title{
On Local One-Particle Approximations and Locally Conserved Currents II
}

\author{
Uta VöLKel and Adolf H. VöLKeL* \\ Department of Physics, University of Pittsburgh \\ Received November 15, 1967
}

\begin{abstract}
As an extension of earlier work a local one-particle approximation is constructed for matrix elements of two four vector currents. The approximation is constructed to fulfill a divergence condition which makes sure, that current conservation is not destroyed by the approximation.
\end{abstract}

\section{Introduction}

This article is in several respects an extension of an earlier paper [1], to which we will refer as I.

In I a "one particle approximation" has been constructed for truncated matrix elements of a vector current and a scalar field, which does not destroy any of the properties following from general principles of local quantum field theory, especially locality and current conservation. This idea was applied [2] to the calculation of mass relations from the "one particle saturation" of SU (3) current algebras, which led to an understanding of the spin dependence of these relations. The structure of the equal time limit in [2] seems to indicate the possibility in our approximation to equal time current commutators to derive relations between different form factors for arbitrary momentum transfer without running into difficulties shown up by other approaches to this problem. To do this we first have to extend the "one particle approximation" of I to the case of matrix elements of two vector currents. This generalization is the main object of the present article.

Usually the local one particle structure of four-point matrix elements is given by products of two three-point matrix elements and a kernel built up from two-point functions, which explicitly contains the singular structure on the mass shell [1]-[8]. In I this singular kernel was built up from the free two-point functions, but from the point of view of perturbation theory it seems more convenient to use the two-point functions of the interacting fields. This generalization will be done in section II for the case of one vector current and one scalar field. The results

* Supported in part by the U.S. Atomic Energy Commission under Contract AT (30-1)-3829.

18 Commun.math. Phys., Vol. 7 
of this section will then be used in section III to construct a local one particle approximation for matrix elements of two vector currents which does not destroy current conservation.

We treat here commutator matrix elements for theories with one scalar particle of mass $m$ only. The generalization to theories with several particles and higher spins as well as the way back to the matrix elements themselves are obvious from I. In order to avoid unnecessary complications we will use the language of generalized functions or simply functions. All our expressions, however, are correct in the sense of continuous linear functionals on some test function space.

\section{Divergence Condition for one Four Vector and One Scalar}

We want to investigate matrix elements of field operators with the usual properties [9], [10]:

\section{Poincaré covariance \\ (A) Locality \\ Spectrum condition \\ Completeness}

About the spectrum of states we assume the existence of a unique invariant vacuum state, a discrete one particle state of mass $m$ and a continuum of many particle states starting at $(2 \mathrm{~m})^{2}$.

Notation: In the following $j^{\mu}, g^{\nu}$ always denote convariant vector fields, $j, g$ scalar fields, especially the divergences of $j^{\mu}$ and $g^{\nu}$ respectively. $B(x)$ denotes a field associated with the particle of mass $m$ of the theory, that means $B(x)$ has a non-vanishing matrix element between the vacuum and the one particle states:

$$
\langle\mathbf{k}, m|\mathrm{~B}(0)| 0\rangle=(2 \pi)^{-3 / 2} .
$$

All fields are assumed to be local and also local relative to each other. In Minkowski space we use the metric $p x=: p^{0} x^{0}-\mathbf{p x}$.

We proceed in two steps. First we write down a covariant local "one particle approximation" for the truncated commutator matrix elements and then we extend this expression without destroying its locality, covariance and spectrum properties in such a way, that current conservation is also valid. By the "one particle approximation" $\left\langle k_{1}\left|j^{\mu}(x) g(y)\right| k_{2}\right\rangle^{I}$ for the truncated matrix elements $\left\langle k_{1}\left|j^{\mu}(x) g(y)\right| k_{2}\right\rangle^{T}$ we mean a sum of products of three-point functions, which is local, covariant, and has the spectrum property:

$$
\begin{aligned}
\left\langle k_{1}\left|\tilde{j}^{\mu}(q) \tilde{g}(p)\right| k_{2}\right\rangle^{T} & =\left\langle k_{1}\left|\tilde{j}^{\mu}(q) \tilde{g}(p)\right| k_{2}\right\rangle^{I} \\
& =\int \frac{d^{3} k}{2 \omega}\left\langle k_{1}\left|\tilde{j}^{\mu}(q)\right| m, \mathbf{k}\right\rangle\left\langle\mathbf{k}, m|\tilde{g}(p)| k_{2}\right\rangle \\
\text { for } 0 & \leqq\left(k_{1}+q\right)^{2}<4 m^{2} ; \omega=:+\sqrt{m^{2}+\mathbf{k}^{2}} .
\end{aligned}
$$


In order to fulfill locality we are forced to add further contributions from the many particle intermediate states to the simple term (2). All these contributions must vanish in the above region. $0 \leqq\left(k_{1}+q\right)^{2}<4 m^{2}$.

A suitable expression for the corresponding commutator matrix elements, which fulfills these conditions is given by [1]:

$\left\langle k_{1}\left|\left[j^{\mu}(x), g(y)\right]\right| k_{2}\right\rangle^{I}=2 \pi i \int d^{4} q$

$$
\begin{aligned}
& \cdot\left\{\left[\left\langle k_{1}|\mathfrak{R}[g(0), \widetilde{\mathrm{B}}(-q)]| 0\right\rangle \overleftrightarrow{\Delta}_{\mathrm{ret}}^{+}(q)\left\langle 0\left|\mathfrak{R}\left[\tilde{\mathrm{B}}(q), j^{\mu}(0)\right]\right| k_{2}\right\rangle\right.\right. \\
& \left.-\left\langle k_{1}|\mathfrak{R}[\widetilde{\mathrm{B}}(-q), g(0)]| 0\right\rangle \overleftrightarrow{\Delta}_{\mathrm{av}}^{\prime}(q)\left\langle 0\left|\mathfrak{R}\left[j^{\mu}(0), \widetilde{\mathrm{B}}(q)\right]\right| k_{2}\right\rangle\right] \\
& \cdot e^{-i\left(k_{2}-q\right) x} e^{i\left(k_{1}-q\right) y} \\
& -\left[\left\langle k_{1}\left|\mathfrak{R}\left[j^{\mu}(0), \widetilde{\mathrm{B}}(-q)\right]\right| 0\right\rangle \overleftrightarrow{\Delta}_{\mathrm{ret}}^{\prime}(q)\left\langle 0|\mathfrak{R}[\widetilde{\mathrm{B}}(q), g(0)]| k_{2}\right\rangle\right. \\
& \left.-\left\langle k_{1}\left|\mathfrak{R}\left[\widetilde{\mathrm{B}}(-q), j^{\mu}(0)\right]\right| 0\right\rangle \overleftrightarrow{\Delta}_{\mathrm{av}}^{\prime}(q)\left\langle 0|\mathfrak{R}[g(0), \widetilde{\mathrm{B}}(q)]| k_{2}\right\rangle\right] \\
& \left.-e^{i\left(k_{1}-q\right) x} e^{-i\left(k_{2}-q\right) y}\right\} .
\end{aligned}
$$

The retarded and advanced matrix elements

$$
\begin{aligned}
\langle|\mathfrak{R}[g(0), \widetilde{\mathrm{B}}(-q)]|\rangle & -\langle|\mathfrak{R}[\widetilde{\mathrm{B}}(-q), g(0)]|\rangle \\
& =i\langle|[\tilde{\mathrm{B}}(-q), g(0)]|\rangle
\end{aligned}
$$

are defined by Jost-Lehmann-Dyson representations [11-13]. In contrast to [1] the kernel

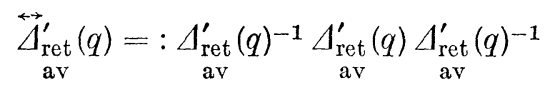

now is given by the two-point functions of the interacting field $B(x)$. They can be represented by $[5,6]$ :

$$
\begin{gathered}
\Delta_{\mathrm{rat}}^{\prime}(q)=\frac{1}{m^{2}-(q \pm i \varepsilon)^{2}}+\int_{4 m^{2}}^{\infty} \frac{d s \varrho(s)}{s-(q \pm i \varepsilon)^{2}}, \\
\Delta_{\substack{\mathrm{av} \\
\mathrm{av}}}^{\prime}(q)^{-1}=\left(m^{2}-q^{2}\right)\left\{A+\sum_{v} \frac{C_{\nu}}{\left[m_{\nu}^{2}-(q \pm i \varepsilon)^{2}\right]\left[m_{\nu}^{2}-m^{2}\right]}\right. \\
\left.+\int_{4 m^{2}}^{\infty} \frac{d s \bar{\varrho}(s)}{\left[s-(q \pm i \varepsilon)^{2}\right]\left[s-m^{2}\right]}\right\} . \\
A \geqq 0, C_{v} \geqq 0, m^{2}<m_{0}^{2}<4 m^{2}, m_{v}^{2}>4 m^{2}, \quad v=1,2, \ldots \\
\varrho(s) \geqq 0, \varrho(s) \geqq 0 .
\end{gathered}
$$

If $j^{\mu}(x)$ is a conserved current the following relation holds for the complete matrix elements :

$$
\begin{aligned}
\partial_{(x) \mu}\left\langle\left|\left[j^{\mu}(x), g(y)\right]\right|\right\rangle & =\left\langle\left|\left[\partial_{\mu} j^{\mu}(x), g(y)\right]\right|\right\rangle=0, \\
\partial_{(x) \mu} & =: \frac{\partial}{\partial x^{\mu}} .
\end{aligned}
$$

The left hand part of this equation also holds in the case of a non conserved current. These properties should also be valid in any reasonable approximation to the matrix elements. 
Because of the occurrence of the retarded and advanced functions, however, we obtain from (3):

$$
\begin{aligned}
\partial_{(x) \mu}\left\langle k_{1}\right| & {\left[j^{\mu}(x), g(y)\right]\left|k_{2}\right\rangle^{I}-\left\langle k_{1}\left|\left[\partial_{\mu} j^{\mu}(x), g(y)\right]\right| k_{2}\right\rangle^{I} } \\
= & 2 \pi e^{i\left(k_{1} x-k_{2} y\right)} \int d^{4} q e^{-i q(x-y)} \mathfrak{P}_{j}\left(k_{1}, q\right)_{1} \\
& \cdot\left[\overleftrightarrow{\Delta}_{\mathrm{ret}}^{\prime}(q)\left\langle 0|\mathfrak{R}[\widetilde{\mathrm{B}}(q), g(0)]| k_{2}\right\rangle-\overleftrightarrow{\Delta}_{\mathrm{av}}^{\prime}(q)\left\langle 0|\Re[g(0), \widetilde{\mathrm{B}}(q)]| k_{2}\right\rangle\right] \\
& +2 \pi e^{-i\left(k_{2} x-k_{1} y\right)} \int d^{4} q e^{i q(x-y)} \mathfrak{P}_{j}\left(k_{2}, q\right)_{2} \\
& \cdot\left[\left\langle k_{1}|\mathfrak{R}[g(0), \widetilde{\mathrm{B}}(-q)]| 0\right\rangle{\overleftrightarrow{\Delta_{\mathrm{ret}}^{\prime}}}^{\prime}(q)\right. \\
& \left.-\left\langle k_{1}|\mathfrak{R}[\widetilde{\mathrm{B}}(-q), g(0)]| 0\right\rangle{\overleftrightarrow{\Delta_{\mathrm{av}}}}_{\mathrm{av}}^{\prime}(q)\right],
\end{aligned}
$$

with the abbreviations:

$$
\begin{aligned}
\mathfrak{P}_{j}\left(k_{1}, q\right)_{1}= & :\left(k_{1}-q\right)_{\mu}\left\langle k_{1}\left|\mathfrak{R}\left[j^{\mu}(0), \widetilde{\mathrm{B}}(-q)\right]\right| 0\right\rangle \\
& +i\left\langle k_{1}\left|\mathfrak{R}\left[\partial_{\mu} j^{\mu}(0), \widetilde{\mathrm{B}}(-q)\right]\right| 0\right\rangle \\
\mathfrak{P}_{j}\left(k_{2}, q\right)_{2}= & :\left(k_{2}-q\right)_{\mu}\left\langle 0\left|\mathfrak{R}\left[\tilde{\mathrm{B}}(q), j^{\mu}(0)\right]\right| k_{2}\right\rangle \\
& -i\left\langle 0\left|\mathfrak{R}\left[\tilde{\mathrm{B}}(q), \partial_{\mu} j^{\mu}(0)\right]\right| k_{2}\right\rangle .
\end{aligned}
$$

Locality implies the functions $\mathfrak{P}_{j}\left(k_{r}, q\right)_{r}$ to be polynomials in $q$. In order to save the property (8) we are forced to extend the approximation (3) adding further terms, such that the new expression

$$
\left\langle\left|\left[j^{\mu}(x), g(y)\right]\right|\right\rangle^{G}=:\left\langle\left|\left[j^{\mu}(x), g(y)\right]\right|\right\rangle^{I}-\mathrm{K}_{g}^{\mu}(x, y)
$$

satisfies the following conditions

$$
\begin{aligned}
\partial_{(x) \mu}\left\langle\left|\left[j^{\mu}(x), g(y)\right]\right|\right\rangle^{G} & =\left\langle\left|\left[\partial_{\mu} j^{\mu}(x), g(y)\right]\right|\right\rangle^{G} \\
& \equiv\left\langle\left|\left[\partial_{\mu} j^{\mu}(x), g(y)\right]\right|\right\rangle^{I} .
\end{aligned}
$$

Furthermore, the new approximation must have the same locality and spectrum properties as the original one. This leads to a boundary value problem for $\mathrm{K}_{g}^{\mu}$.

According to the structure of the right hand sides of the Eqs. (3) and (9), we split $\mathrm{K}_{g}^{\mu}$ into two parts:

$$
\mathrm{K}_{g}^{\mu}(x, y)=e^{i\left(k_{1} x-k_{2} y\right)} \mathrm{K}_{g}^{\mu}(x-y)_{1}-e^{-i\left(k_{2} x-k_{1} y\right)} \mathrm{K}_{g}^{\mu}(x-y)_{2} .
$$

Defining the fourier transforms $\tilde{\mathrm{K}}_{g}^{\mu}(q)$, by

$$
\widetilde{\mathrm{K}}_{g}^{\mu}(q)_{2}=: \frac{1}{(2 \pi)^{5 / 2}} \int d^{4} x e^{ \pm i q x} \mathrm{~K}_{g}^{\mu}(x)_{2},
$$

from the Eqs. (3), (9)-(13) we obtain the following boundary value problem for $\widetilde{\mathrm{K}}_{g}^{\mu}(q)_{r}$ :

$$
\begin{gathered}
\left(q-k_{1}\right)_{\mu} \tilde{\mathrm{K}}_{g}^{\mu}(q)_{1}=\tilde{\mathrm{I}}_{g}(q)_{1} \\
\left(q-k_{2}\right)_{\mu} \tilde{\mathrm{K}}_{g}^{\mu}(q)_{2}=\tilde{\mathbf{I}}_{g}(q)_{2}, \\
\mathrm{~K}_{g}^{\mu}(x)_{r}=0 \text { for } \quad x^{2}<0 \\
\operatorname{supp}\left\{\tilde{\mathrm{K}}_{g}^{\mu}(q)_{r}\right\} \cong \operatorname{supp}\left\{\tilde{\mathrm{I}}_{g}(q)_{r}\right\}, \quad r=1,2
\end{gathered}
$$


where the right hand sides of (14) are explicitly given by:

$$
\begin{aligned}
\tilde{\mathrm{I}}_{g}(q)_{1}= & : i(2 \pi)^{5 / 2} \mathfrak{P}_{j}\left(k_{1}, q\right)_{1}\left[\overleftrightarrow{\Delta}_{\mathrm{ret}}^{\prime}(q)\left\langle 0|\mathfrak{R}[\widetilde{\mathrm{B}}(q), g(0)]| k_{2}\right\rangle\right. \\
& \left.-{\overleftrightarrow{\Delta_{\mathrm{av}}^{\prime}}}^{\prime}(q)\left\langle 0|\mathfrak{R}[g(0), \widetilde{\mathrm{B}}(q)]| k_{2}\right\rangle\right], \\
\tilde{\mathrm{I}}_{g}(q)_{2}= & : i(2 \pi)^{5 / 2}\left[\left\langle k_{1}|\Re[g(0), \widetilde{\mathrm{B}}(-q)]| 0\right\rangle \overleftrightarrow{\Delta}_{\mathrm{ret}}^{\prime}(q)\right. \\
& \left.-\left\langle k_{1}|\mathfrak{R}[\widetilde{\mathrm{B}}(-q), g(0)]| 0\right\rangle \overleftrightarrow{\Delta}_{\mathrm{av}}^{\prime}(q)\right] \mathfrak{P}_{j}\left(k_{2}, q\right)_{2} .
\end{aligned}
$$

For the case of a symmetric mass spectrum the most general solutions of these boundary value problems have been constructed in I by means of Jost-Lehmann representations $[11,13]$. For the case of non symmetric mass spectrum a class of solutions has been constructed using Dyson representations $[12,13]$. This class is not the most general one, because the Dyson spectral functions are not unique. We now construct the most general solution of (14), (15).

The inhomogeneous parts of (14) have all the properties of commutator matrix elements as follows from (16), (17). Therefore their Dyson representations are:

$$
\tilde{\mathrm{I}}_{g}(q)_{r}=\int d^{4} u d s \varepsilon\left(q^{0}-u^{0}\right) \delta\left((u-q)^{2}-s\right) \phi_{g}(u, s)_{r} .
$$

The spectral functions $\phi_{g}$ have the well known support properties $[12,13]$, which automatically guarantee the boundary conditions $(15)^{1}$.

For $\widetilde{\mathrm{K}}_{g}^{\mu}$ we make the ansatz:

$$
\begin{aligned}
\tilde{K}_{g}^{\mu}(q)_{r}= & \int d^{4} u d s \varepsilon\left(q^{0}-u^{0}\right) \delta\left((q-u)^{2}-s\right) \\
& \cdot\left\{\left(q+k_{r}-2 u\right)^{\mu} \Phi_{g}(u, s)_{r}-\frac{\partial}{\partial s} Z_{g}^{\mu}(u, s)_{r}\right\},
\end{aligned}
$$

with arbitrary Dyson spectral functions $\Phi_{g}, \mathrm{Z}_{g}^{\mu}$. By means of the identity $\int d^{4} u d s \varepsilon\left(q^{0}-u^{0}\right) \delta\left((q-u)^{2}-s\right)\left\{(q-u)_{\mu} \frac{\partial}{\partial s}-\frac{1}{2} \partial_{(u) \mu}\right\} \psi(u, s)=0$

it follows from (14):

$$
\begin{aligned}
(s-(u & \left.\left.-k_{r}\right)^{2}\right) \Phi_{g}(u, s)_{r}-\phi_{g}(u, s)_{r}-\varrho_{g}(u, s)_{r} \\
- & -\left[\left(u-k_{r}\right)_{\mu} \frac{\partial}{\partial s}+\frac{1}{2} \partial_{(u) \mu}\right] Z_{g}^{\mu}(u, s)_{r}=0 .
\end{aligned}
$$

Here $\varrho_{g}(u, s)_{r}$ are arbitrary Dyson spectral functions with

$$
\int d^{4} u d s \varepsilon\left(q^{0}-u^{0}\right) \delta\left((q-u)^{2}-s\right) \varrho_{g}(u, s)_{r}=0 .
$$

They appear in (21) because of the nonuniqueness of the Dyson representation.

1 In the following by arbitrary Dyson spectral functions we mean spectral functions with these support properties. 
Solving Eq. (21) for $\Phi_{g}(u, s)_{r}$ we obtain the most general solution of the boundary value problem (14), (15):

$$
\begin{aligned}
\tilde{\mathrm{K}}_{g}^{\mu}(q)_{r} & =\int d^{4} u d s \varepsilon\left(q^{0}-u^{0}\right) \delta\left((q-u)^{2}-s\right) \\
& \cdot\left\{\frac { ( q + k _ { r } - 2 u ) ^ { \mu } } { s - ( u - k _ { r } ) ^ { 2 } } \left[\phi_{g}(u, s)_{r}+\varrho_{g}(u, s)_{r}\right.\right. \\
+ & \left.\left(\left(u-k_{r}\right)_{v} \frac{\partial}{\partial s}+\frac{1}{2} \partial_{(u) v}\right) \mathrm{Z}_{g}^{v}(u, s)_{r}\right]-\frac{\partial}{\partial s} \mathrm{Z}_{g}^{\mu}(u, s)_{r} \\
+ & \left.\left(q+k_{r}-2 u\right)^{\mu} \delta\left(s-\left(u-k_{r}\right)^{2}\right) \mathrm{E}_{g}(u, s)_{r}\right\} .
\end{aligned}
$$

$\mathrm{E}_{g}(u, s)_{r}$ is the extension in the sense of SchwaRTz [14] of an arbitrary Dyson spectral function given on the regular surface $s-\left(u-k_{r}\right)^{2}=0$.

Remark. The most general solution (23) differs from those constructed in I only by the additional term $\varrho_{g}$. Because of Eq. (22) this term cannot contribute to the equal time limit, provided it has a finite limit. Therefore, the applications discussed in [2] are independent of this term.

For the considerations in the next section it is more suitable to write the solution (23) in the following form, which one obtains immediately by means of (20) and some elementary calculations:

$$
\begin{aligned}
\tilde{\mathrm{K}}_{g}^{\mu}(q)_{r} & =\int d^{4} u d s \varepsilon\left(q^{0}-u^{0}\right) \delta\left((q-u)^{2}-s\right) \\
& \cdot\left\{\frac{\left(q+k_{r}-2 u\right)^{\mu}}{s-\left(u-k_{r}\right)^{2}}\left[\phi_{g}(u, s)_{r}+\varrho_{g}(u, s)_{r}+\left(q-k_{r}\right)_{v} \mathrm{~W}_{g}^{v}(u, s)_{r}\right]\right. \\
& \left.-\mathrm{W}_{g}^{\mu}(u, s)_{r}+\left(q+k_{r}-2 u\right)^{\mu} \delta\left(s-\left(u-k_{r}\right)^{2}\right) \mathrm{E}_{g}(u, s)_{r}\right\}
\end{aligned}
$$

with

$$
\frac{W_{g}^{\mu}(u, s)_{r}}{s-\left(u-k_{r}\right)^{2}}=: \frac{\partial}{\partial s} \frac{Z_{g}^{\mu}(u, s)_{r}}{s-\left(u-k_{r}\right)^{2}} .
$$

\section{Divergence Condition for Two Vector Currents}

In analogy to the case of one vector current and one scalar field we must extend the "one particle approximation" for matrix elements of two vector currents $\left\langle\left|\left[j^{\mu}, g^{v}\right]\right|\right\rangle^{I}$ adding further terms in such a way, that the new matrix element

$$
\left\langle\left|\left[j^{\mu}(x), g^{v}(y)\right]\right|\right\rangle^{G}=:\left\langle\left|\left[j^{\mu}(x), g^{v}(y)\right]\right|\right\rangle^{I}-\mathrm{K}^{\mu \nu}(x, y),
$$

satisfies the following two divergence conditions:

$$
\begin{aligned}
\partial_{(x) \mu}\left\langle\left|\left[j^{\mu}(x), g^{v}(y)\right]\right|\right\rangle^{G} & =\left\langle\left|\left[\partial_{\mu} j^{\mu}(x), g^{v}(y)\right]\right|\right\rangle^{G} \\
& \equiv\left\langle\left|\left[\partial_{\mu} j^{\mu}(x), g^{v}(y)\right]\right|\right\rangle^{I}-\mathrm{K}_{j}^{v}(x, y), \\
\partial_{(y) \nu}\left\langle\left|\left[j^{\mu}(x), g^{\nu}(y)\right]\right|\right\rangle^{G} & =\left\langle\left|\left[j^{\mu}(x), \partial_{\nu} g^{v}(y)\right]\right|\right\rangle^{G} \\
& \equiv\left\langle\left|\left[j^{\mu}(x), \partial_{\nu} g^{\nu}(y)\right]\right|\right\rangle^{I}-\mathrm{K}_{g}^{\mu}(x, y) .
\end{aligned}
$$

From these two equations it follows by means of Eq. (11):

$$
\partial_{(x) \mu} \partial_{(y) \nu}\left\langle\left|\left[j^{\mu}(x), g^{\nu}(y)\right]\right|\right\rangle^{G}=\left\langle\left|\left[\partial_{\mu} j^{\mu}(x), \partial_{\nu} g^{\nu}(y)\right]\right|\right\rangle^{I} \text {. }
$$

$\mathrm{K}_{g}^{\mu}(x, y)$ is given by (12), (13) and $(24)^{2}$. Similarly $\mathrm{K}_{j}^{v}(x, y)$ has the

${ }^{2}$ From now on $j(x), g(y)$ are identical to the divergence of $j^{\mu}(x)$ and $g^{v}(y)$ respectively. $j(x)=\partial_{\mu} j^{\mu}(x), g(y)=\partial_{\nu} g^{\nu}(y)$. 
representation:

$$
\begin{aligned}
& \tilde{\mathrm{K}}_{j}^{v}(q)_{r}=\int d^{4} u d s \varepsilon\left(q^{0}-u^{0}\right) \delta\left((q-u)^{2}-s\right) \\
& \cdot\left\{\frac{\left(q+k_{3-r}-2 u\right)^{\nu}}{s-\left(u-k_{3-r}\right)^{2}}\left[\phi_{j}(u, s)_{r}+\varrho_{j}(u, s)_{r}+\left(q-k_{3-r}\right)_{\mu} \mathrm{W}_{j}^{\mu}(u, s)_{r}\right]\right. \\
& \left.-\mathrm{W}_{j}^{v}(u, s)_{r}+\left(q+k_{3-r}-2 u\right)^{\nu} \delta\left(s-\left(u-k_{3-r}\right)^{2}\right) \mathrm{E}_{j}(u, s)_{r}\right\}
\end{aligned}
$$

$\phi_{j}(u, s)_{r}$ are the Dyson spectral functions of the following (commutator) matrix elements :

$$
\begin{aligned}
\tilde{\mathrm{I}}_{j}(q)_{1}= & : i(2 \pi)^{5 / 2}\left\{\left\langle k_{1}\left|\mathfrak{R}\left[\partial_{\mu} j^{\mu}(0), \tilde{\mathrm{B}}(-q)\right]\right| 0\right\rangle \overleftrightarrow{\Delta}_{\mathrm{ret}}^{\prime}(q)\right. \\
& \left.-\left\langle k_{1}\left|\mathfrak{R}\left[\tilde{\mathrm{B}}(-q), \partial_{\mu} j^{\mu}(0)\right]\right| 0\right\rangle \overleftrightarrow{\Delta}_{\mathrm{av}}^{\prime}(q)\right\} \mathfrak{P}_{g}\left(k_{2}, q\right)_{2}, \\
\tilde{\mathrm{I}}_{j}(q)_{2}= & : i(2 \pi)^{5 / 2} \mathfrak{P}_{g}\left(k_{1}, q\right)_{1} \overleftrightarrow{\Delta}_{\mathrm{ret}}^{\prime}(q)\left\langle 0\left|\mathfrak{R}\left[\tilde{\mathrm{B}}(q), \partial_{\mu} j^{\mu}(0)\right]\right| k_{2}\right\rangle \\
& \left.-\overleftrightarrow{\Delta}_{\mathrm{av}}^{\prime}(q)\left\langle 0\left|\mathfrak{R}\left[\partial_{\mu} j^{\mu}(0), \tilde{\mathrm{B}}(q)\right]\right| k_{2}\right\rangle\right\},
\end{aligned}
$$

where the polynomials $\mathfrak{P}_{g}$ are given by $(10)$ with $j$ replaced by $g$.

If we split $K^{\mu \nu}(x, y)$ in the manner of Eq. (12) then by means of (3), (26)-(28) we arrive at the following two boundary value problems for its fourier transforms $\widetilde{\mathrm{K}}^{\mu \nu}(q)_{r}$ :

$$
\begin{aligned}
\left(q-k_{1}\right)_{\mu} \tilde{\mathrm{K}}^{\mu \nu}(q)_{1} & =i \tilde{\mathrm{K}}_{j}^{\nu}(q)_{1}+\tilde{\mathrm{I}}_{g}^{\nu}(q)_{1} \\
\left(q-k_{2}\right)_{\nu} \tilde{\mathrm{K}}^{\mu \nu}(q)_{1} & =-i \tilde{\mathrm{K}}_{g}^{\mu}(q)_{1}+\tilde{\mathrm{I}}_{j}^{\mu}(q)_{1}, \\
\left(q-k_{2}\right)_{\mu} \tilde{\mathrm{K}}^{\mu \nu}(q)_{2} & =-i \tilde{\mathrm{K}}_{j}^{v}(q)_{2}+\tilde{\mathrm{I}}_{g}^{v}(q)_{2} \\
\left(q-k_{1}\right)_{v} \tilde{\mathrm{K}}^{\mu \nu}(q)_{2} & =i \tilde{\mathrm{K}}_{g}^{\mu}(q)_{2}+\tilde{\mathrm{I}}_{j}^{\mu}(q)_{2}, \\
\mathrm{~K}^{\mu \nu}(x)_{r} & =0 \text { for } x^{2}<0
\end{aligned}
$$

$\operatorname{supp}\left\{\tilde{\mathrm{K}}^{\mu \nu}(q)_{r}\right\} \leqq \operatorname{supp}\{$ inhomogeneous terms $\}, r=1,2$.

On the right hand side of (33) and (34) the functions $\tilde{I}_{g}^{\nu}(q)_{r}$ and $\tilde{I}_{j}^{\mu}(q)_{r}$ are defined by $\left(16,(17)\right.$ replacing $g(0)$ by $g^{\nu}(0)$ and by $(31),(32)$ replacing $\partial_{\mu} j^{\mu}(0)$ by $j^{\mu}(0)$, respectively.

Again we solve the problems by means of Dyson representations [12, 13] which automatically contain the boundary conditions (35) in the form of support properties of the integral kernels.

In order to do this we have to introduce spectral functions for the commutators $\tilde{\mathrm{I}}^{v}(q)$. These commutators however are not independent of $\tilde{\mathrm{I}}(q)$. Roughly speaking $\tilde{\mathrm{I}}(q)$ is the divergence of $\tilde{\mathrm{I}}^{v}(q)$. Corresponding to this the spectral functions of $\tilde{\mathrm{I}}^{v}(q)$ will be connected to the spectral functions $\phi(u, s)$ of $\tilde{\mathrm{I}}(q)$. 
From the Eqs. (10), (16), (17), (31), (32) we obtain the connection between $\tilde{\mathrm{I}}^{v}(q)$ and $\tilde{\mathrm{I}}(q)$ :

$$
\begin{aligned}
\left(q-k_{r}\right)_{\mu} \tilde{\mathrm{I}}_{j}^{\mu}(q)_{r}= & -i(-1)^{r}\left\{\tilde{\mathrm{I}}_{j}(q)_{r}\right. \\
& +(2 \pi)^{5 / 2}(-1)^{r} \mathfrak{P}_{j}\left(k_{r}, q\right)_{r}\left[\overleftrightarrow{\Delta}_{\mathrm{ret}}^{\prime}(q)\right. \\
& \left.\left.-\overleftrightarrow{\Delta}_{\mathrm{av}}^{\prime}(q)\right] \mathfrak{P}_{g}\left(k_{3-r}, q\right)_{3-r}\right\}, \\
\left(q-k_{3-r}\right)_{\nu} \tilde{\mathrm{I}}_{g}^{v}(q)_{r}= & i(-1)^{r}\left\{\tilde{\mathrm{I}}_{g}(q)_{r}\right. \\
& -(2 \pi)^{5 / 2}(-1)^{r} \mathfrak{P}_{j}\left(k_{r}, q\right)_{r}\left[\overleftrightarrow{\Delta}_{\mathrm{ret}}^{\prime}(q)\right. \\
& \left.\left.-\overleftrightarrow{\Delta}_{\mathrm{av}}^{\prime}(q)\right] \mathfrak{P}_{g}\left(k_{3-r}, q\right)_{3-r}\right\} .
\end{aligned}
$$

By constructing the most general solutions of (36) and (37) we get a Dyson representation of $\tilde{\mathrm{I}}^{v}(q)$ which contains the spectral functions $\phi(u, s)$ together with new functions $\phi^{v}(u, s)$ independent of $\phi(u, \mathrm{~s})$, thus showing explicitly the connection between $\tilde{\mathrm{I}}^{v}(q)$ and $\tilde{\mathrm{I}}(q)$. Let $\pi(u, s)_{r}$ be the Dyson spectral functions ${ }^{3}$ of the second terms in the wavy brackets on the right hand sides of (36), (37). Then according to section II we obtain from (36) and (37) respectively:

$$
\begin{aligned}
\tilde{\mathrm{I}}_{j}^{\mu}(q)_{r}= & -i(-1)^{r} \int d^{4} u d s \varepsilon\left(q^{0}-u^{0}\right) \delta\left((q-u)^{2}-s\right) \\
& \cdot\left\{\frac { ( q + k _ { r } - 2 u ) ^ { \mu } } { s - ( u - k _ { r } ) ^ { 2 } } \left[\phi_{j}(u, s)_{r}+\pi(u, s)_{r}+\psi_{j}(u, s)_{r}\right.\right. \\
& \left.+\left(q-k_{r}\right)_{\lambda} \phi_{j}^{\lambda}(u, s)_{r}\right]-\phi_{j}^{\mu}(u, s)_{r} \\
& \left.+\left(q+k_{r}-2 u\right)^{\mu} \delta\left(s-\left(u-k_{r}\right)^{2}\right) e_{j}(u, s)_{r}\right\} \\
\tilde{\mathrm{I}}_{g}^{v}(q)_{r}= & i(-1)^{r} \int d^{4} u d s \varepsilon\left(q^{0}-u^{0}\right) \delta\left((q-u)^{2}-s\right) \\
& +\left\{\frac { ( q + k _ { 3 - r } - 2 u ) ^ { v } } { s - ( u - k _ { 3 - r } ) ^ { 2 } } \left[\phi_{g}(u, s)_{r}-\pi(u, s)_{r}+\psi_{g}(u, s)_{r}\right.\right. \\
& \left.+\left(q-k_{3-r}\right)_{\lambda} \phi_{g}^{\lambda}(u, s)_{r}\right]-\phi_{g}^{v}(u, s)_{r} \\
& \left.+\left(q+k_{3-r}-2 u\right)^{\nu} \delta\left(s-\left(u-k_{3-r}\right)^{2}\right) e_{g}(u, s)_{r}\right\} .
\end{aligned}
$$

The spectral functions $\psi_{j}, \psi_{g}$ have to satisfy the condition (22).

We want to remark that in contrast to the arbitrary spectral functions $\mathrm{W}^{\mu}, \varrho, \mathrm{E}$, which occurred in (24) section II, the spectral functions $\phi^{\lambda}, \psi, e$ of (38), (39) are not arbitrary. They are the Dyson spectral functions of given matrix elements.

This form of the representation is of great advantage for all applications (for instance equal time commutator algebras), for which one has to make further assumptions on the matrix elements and therewith on the spectral functions. We are allowed to make assumptions for $\phi, \phi^{v}, \psi$, and $e$ independently without running into contradictions to general principles.

3 The support of the spectral functions is concentrated in the point $u=0$ because of the equations (6), (7) and the polynomial character of $\mathfrak{P}$ in $q$. 
It can be verified by insertion that the solution of $(33)-(35)$ is given by

$$
\begin{aligned}
\tilde{\mathrm{K}}^{\mu \nu}(q)_{r} & =i(-1)^{r} \int d^{4} u d s \varepsilon\left(q^{0}-u^{0}\right) \delta\left((q-u)^{2}-s\right) \\
& \cdot\left\{\frac { ( q + k _ { r } - 2 u ) ^ { \mu } } { s - ( u - k _ { r } ) ^ { 2 } } \frac { ( q + k _ { 3 - r } - 2 u ) ^ { \nu } } { s - ( u - k _ { 3 - r } ) ^ { 2 } } \left[\phi_{g}(u, s)_{r}-\phi_{j}(u, s)_{r}-\pi(u, s)_{r}\right.\right. \\
& +\left(q-k_{3-r}\right)_{\lambda}\left(\phi_{g}^{\lambda}(u, s)_{r}-W_{j}^{\lambda}(u, s)_{r}\right. \\
& \left.+\frac{\left(k_{r}-k_{3-r}\right)^{\lambda}}{\left(k_{1}-k_{2}\right)^{2}}\left(\psi_{g}(u, s)_{r}-\varrho_{j}(u, s)_{r}\right)\right) \\
& -\left(q-k_{r}\right)_{\lambda}\left(\phi_{j}^{\lambda}(u, s)_{r}-W_{g}^{\lambda}(u, s)_{r}\right. \\
& \left.\left.-\frac{\left(k_{r}-k_{3-r}\right)^{\lambda}}{\left(k_{1}-k_{2}\right)^{2}}\left(\psi_{j}(u, s)_{r}-\varrho_{g}(u, s)_{r}\right)\right)\right] \\
& +\frac{\left(q+k_{3-r}-2 u\right)^{\nu}}{s-\left(u-k_{3-r}\right)^{2}}\left[\phi_{j}^{\mu}(u, s)_{r}-\mathrm{W}_{g}^{\mu}(u, s)_{r}\right. \\
& -\frac{\left(k_{r}-k_{3-r}\right)^{\mu}}{\left(k_{1}-k_{2}\right)^{2}}\left(\psi_{g}(u, s)_{r}-\varrho_{j}(u, s)_{r}+\psi_{j}(u, s)_{r}-\varrho_{g}(u, s)_{r}\right) \\
& \left.-\left(q+k_{r}-2 u\right)^{\mu} \delta\left(s-\left(u-k_{r}\right)^{2}\right)\left(e_{j}(u, s)_{r}-\mathrm{E}_{g}(u, s)_{r}\right)\right] \\
& -\frac{\left(q+k_{r}-2 u\right)^{\mu}}{s-\left(u-k_{r}\right)^{2}}\left[\phi_{g}^{\nu}(u, s)_{r}-\mathrm{W}_{j}^{\nu}(u, s)_{r}\right. \\
& +\frac{\left(k_{r}-k_{3-r}\right)^{\nu}}{\left(k_{1}-k_{2}\right)^{2}}\left(\psi_{g}(u, s)_{r}-\varrho_{j}(u, s)_{r}+\psi_{j}(u, s)_{r}-\varrho_{g}(u, s)_{r}\right) \\
& \left.\left.-\left(q+k_{3-r}-2 u\right)^{\nu} \delta\left(s-\left(u-k_{3-r}\right)^{2}\right)\left(e_{g}(u, s)_{r}-\mathrm{E}_{j}(u, s)_{r}\right)\right]\right\} \\
& +\tilde{\mathrm{H}}^{\mu \nu}(q)_{r} \cdot \\
r= & 1,2 .
\end{aligned}
$$

Here $\tilde{\mathrm{H}}^{\mu \nu}(q)_{r}$ is the solution of the homogeneous problem

$$
\begin{aligned}
\left(q-k_{r}\right)_{\mu} \tilde{\mathrm{H}}^{\mu \nu}(q)_{r} & =0 \\
\left(q-k_{3-r}\right)_{\nu} \tilde{\mathrm{H}}^{\mu \nu}(q)_{r} & =0, \quad r=1,2
\end{aligned}
$$

with the boundary conditions (35). A class of solutions of this homogeneous problem is given by:

$$
\begin{gathered}
\tilde{\mathrm{H}}^{\mu v}(q)_{r}=\int d^{4} u d s \varepsilon\left(q^{0}-u^{0}\right) \delta\left((q-u)^{2}-s\right) \\
\left\{( q + k _ { r } - 2 u ) ^ { \mu } ( q + k _ { 3 - r } - 2 u ) ^ { v } \left[\frac{\left(q-k_{r}\right)_{\lambda}}{s-\left(u-k_{1}\right)^{2}} \frac{\left(q-k_{3-r}\right)_{x}}{s-\left(u-k_{2}\right)^{2}} Z^{\lambda \varkappa}(u, s)_{r}\right.\right. \\
+\delta\left(s-\left(u-k_{1}\right)^{2}\right) \delta\left(s-\left(u-k_{2}\right)^{2}\right) E(u, s)_{r} \\
+\frac{\delta\left(s-\left(u-k_{3-r}\right)^{2}\right)}{s-\left(u-k_{r}\right)^{2}}\left(q-k_{r}\right)_{\lambda} E_{1}^{\lambda}(u, s)_{r} \\
\left.+\frac{\delta\left(s-\left(u-k_{r}\right)^{2}\right)}{s-\left(u-k_{3-r}\right)^{2}}\left(q-k_{3-r}\right)_{\varkappa} E_{2}^{\varkappa}(u, s)_{r}\right] \\
-\left(q+k_{3-r}-2 u\right)^{v}\left[\frac{\left(q-k_{3-r}\right)_{\varkappa}}{s-\left(u-k_{3-r}\right)^{2}} Z^{\mu \varkappa}(u, s)_{r}+\delta\left(s-\left(u-k_{3-r}\right)^{2}\right) E_{1}^{\mu}(u, s)_{r}\right] \\
-\left(q+k_{r}-2 u\right)^{\mu}\left[\frac{\left(q-k_{r}\right)_{\lambda}}{s-\left(u-k_{r}\right)^{2}} Z^{\lambda v}(u, s)_{r}+\delta\left(s-\left(u-k_{r}\right)^{2}\right) E_{2}^{v}(u, s)_{r}\right] \\
\left.+Z^{\mu v}(u, s)_{r}\right\} .
\end{gathered}
$$

The functions $Z^{\mu v}(u, s)_{r}$ are arbitrary covariant Dyson spectral functions, $E(u, s)_{r}$ and $E_{i}^{\mu}(u, s)_{r}$ are extensions in the sense of ScHwarTz [14] of 
arbitrary Dyson spectral functions which are given on the following surfaces

and

$$
\left[s-\left(u-k_{1}\right)^{2}=0\right] \cap\left[s-\left(u-k_{2}\right)^{2}=0\right]
$$

respectively.

$$
s-\left(u-k_{j}\right)^{2}=0, \quad j=1,2
$$

\section{Final Remarks}

We have written the solutions in the form of Dyson representations. In all applications at a certain point one has to go back to the matrix elements themselves. Therefore the question arises if it is possible to solve the problem in terms of matrix elements. The answer is yes. For we can solve the problem in exactly the same manner by means of Jost-Lehmann representations [1], and at the end use the well known inversion formulas to reexpress the spectral functions by matrix elements.

In that case, however, all calculation are much more involved and the formulas are much more complicated. Furthermore, in the applications we are interested in at the moment (equal time commutator algebras), it is very easy at a later stage of the calculation to connect the Dyson integrals to matrix elements [2]. Therefore, we preferred to construct the solutions by means of the more compact Dyson representation.

Acknowledgments. We want to thank Profs. B. Schroer and P. STicher for many helpful discussions, for their interest in this work and their constant encouragement.

\section{References}

1. Völkel, A. H.: Commun. Math. Phys. 5, 57 (1967).

2. - Mass relations for particles of arbitrary spin. To be published in Ann. Phys. (N. Y.).

3. Zrmmermann, W.: Nuovo Cimento 13, 503 (1959).

4. - Nuovo Cimento 16, 690 (1960).

5. Symanzik, K.: J. Math. Phys. 1, 249 (1960).

6. - Lectures in theoretical physics. Vol. III. Boulder 1960. New York: Interscience Publishers 1961.

7. Streater, R. F.: Nuovo Cimento 25, 274 (1962).

8. Völkel, A. H.: Commun. Math. Phys. 2, 176 (1966).

9. Streater, R. F., and A. S. Wightman: PCT, spin and statistics and all that. New York: W. A. Benjamin 1964.

10. Jost, R.: The general theory of quantized fields. Providence, Rhode Island: The American Mathematical Society, 1965.

11. Jost, R., and H. Lehmann: Nuovo Cimento 5, 1598 (1957).

12. Dyson, F. J.: Phys. Rev. 110, 1460 (1958).

13. Wightmann, A. S.: In: Dispersion relations and elementary particles. Paris: Hermann 1960.

14. Schwartz, L.: Theorie des distributions I, II. Paris: Hermann 1957 et 1959.

Dr. A. H. VöLKeL

Department of Physics

University of Pittsburgh

Pittsburgh, Pennsylvania 15213, USA 